\title{
The quantum-mechanical position operator and the polarization problem
}

\author{
Raffaele Resta \\ INFM - Dipartimento di Fisica Teorica, \\ Università di Trieste, Strada Costiera 11, I-34014 Trieste, Italy \\ and \\ Department of Physics, The Catholic University of America, Washington, D.C. 20064
}

\begin{abstract}
The position operator (defined within Schrödinger representation as usual) becomes meaningless when the usual Born-von Kármán periodic boundary conditions are adopted: this fact is at the root of the polarization problem. I show how to define the position expectation value by means of rather peculiar many-body (multiplicative) operator acting on the wavefunction of the extended system. This definition can be regarded as the generalization of a precursor work, apparently unrelated to the polarization problem. For uncorrelated electrons, the present finding coincides with the so-called "single-point Berry phase" formula, which can hardly be regarded as the approximation of a continuum integral, and is computationally very useful for disordered systems. Simulations which are based on this concept are being performed by several groups.
\end{abstract}

\section{INTRODUCTION}

In a phenomenological description of dielectric media the concept of macroscopic polarization is the basic one [1]. One formally defines the polarization of a macroscopic sample as its electrical dipole moment, divided by the volume. The problem is that the dipole of a finite system is dominated by surface effects, which seem to make polarization ill defined as a property of the bulk. In condensed matter theory, one typically gets rid of undesirable surface effects by dealing with extended systems within Born-von Kármán periodic boundary conditions (BvK). Unfortunately, this does not solve the polarization problem: the dipole is in fact the expectation value of the quantum-mechanical electronic position, which in its usual form is an ill defined operator within BvK.

Because of the above reasons, even defining what bulk dielectric polarization is remained a major challenge for many years. If one assumes the polarization elements as discrete, à la Clausius-Mossotti, then the polarization mechanism can be safely understood: but such oversimplified picture does not apply to a real dielectric, where the electronic distribution is continuous, and often very delocalized. 
Typically, textbooks attempt to explain polarization of a bulk periodic solid via the dipole moment of a unit cell, or something of the kind $[2,3]$. These definitions are incorrect [4]: according to the modern viewpoint, bulk macroscopic polarization is a physical observable completely independent from the periodic charge distribution of the polarized dielectric.

The breakthrough was fostered by the 1992 Williamsburg meeting. Macroscopic polarization was defined in terms of the wavefunctions, not of the charge. This definition has an unambiguous thermodynamic limit, such that BvK and Bloch states can be used with no harm [5]. In the following months a modern theory of macroscopic polarization in crystalline dielectrics has been completely established [6,7], thanks to a major advance due to R.D. King-Smith and D. Vanderbilt [8], who expressed polarization in terms of a Berry phase $[9,10]$. An early comprehensive account of the modern theory exists [7]. Other less technical presentations are available as well [11,12]; for an oversimplified outline see Ref. [13].

In its original form, the Berry-phase theory of polarization was based on independent-electron wavefunctions, such as the Kohn-Sham orbitals of densityfunctional theory $[7,14]$. Its generalization to correlated many-electron wavefunctions is due to Ortíz and Martin [15]. Both the independent-electron and the correlated-electron versions of the Berry-phase theory of polarization rely on some lattice periodicity, and define macroscopic polarization in the form of a reciprocalspace integral. In particular, the Ortíz-Martin polarization of a correlated manyelectron system takes the form of a peculiar "ensemble average", since it is an integral over a set of many-body ground states. This viewpoint is indeed correct and very valuable: the Ortíz-Martin theory has even been implemented to study the polarization of interesting model systems of highly correlated electrons [16-18]. However, the fact that macroscopic polarization could not be even defined by means a "pure state" expectation value (over a single many-electron ground state) was a disturbing drawback. This has been overcome thanks to the advance of Ref. [19], where a novel solution to the polarization problem is provided. The compact formula arrived at in Ref. [19], Eq. (14) below, is apparently unrelated to the Berry-phase concept; it applies on the same footing to correlated systems and to independent-electron systems, as well as to crystalline and to disordered systems. Lattice periodicity and integration in reciprocal space are no longer needed in order to define what polarization is. Polarization can in fact be cast as the expectation value of a rather peculiar operator over the many-electron ground wavefunction (in the thermodynamic limit).

In the present work, I present the main achievement of Ref. [19] under a rather different light than in the original paper. I will show that its main result can be regarded as the many-electron generalization of a precursor work, apparently unrelated to the polarization problem [20]. I will also discuss in detail the independentelectron case, linking in particular the results of Ref. [19] to the so-called singlepoint Berry phase [21,22], a concept which proves very useful, particularly in the study of disordered systems. 


\section{THE HILBERT SPACE OF CONDENSED MATTER}

Almost invariably, in condensed matter theory one adopts BvK for the electronic wavefunctions, when dealing with either crystalline systems or disordered systems. There are several good reasons for such choice. One reason is that one eliminates any surface by construction, thus getting rid of indesirable surface effects. Another reason is that in the crystalline case one can exploit the virtues of Bloch's theorem. I stress that the BvK choice is tantamount to defining the Hilbert space where our solutions of Schrödinger equation live.

For the sake of simplicity, the present work will deal with the one-dimensional case. For a single-particle wavefunction BvK reads $\psi(x+L)=\psi(x)$, where $L$ is the imposed periodicity, chosen to be large with respect to atomic dimensions. Notice that lattice periodicity is not assumed, and BvK applies to disordered systems as well. An operator maps any vector of the given Hilbert space into another vector belonging to the same space: the multiplicative position operator $x$ is not a legitimate operator when $\mathrm{BvK}$ are adopted for the state vectors, since $x \psi(x)$ is not a periodic function whenever $\psi(x)$ is such. Of course, any periodic function of $x$ is a legitimate multiplicative operator: this is the case e.g. of the nuclear potential acting on the electrons.

In the following, we will need to explicitly introduce many-body wavefunctions as well. BvK then imposes periodicity in each electronic variable separately:

$$
\Psi_{0}\left(x_{1}, \ldots, x_{i}, \ldots, x_{N}\right)=\Psi_{0}\left(x_{1}, \ldots, x_{i}+L, \ldots, x_{N}\right) .
$$

Our interest is indeed in studying a bulk system: $N$ electrons in a segment of length $L$. Eventually the thermodynamic limit is taken: $L \rightarrow \infty, N \rightarrow \infty$, and $N / L=n_{0}$ constant. We will also assume throughout the ground state nondegenerate, and we deal with insulating systems only: this means that the gap between the ground eigenvalue and the excited ones remains finite for $L \rightarrow \infty$.

In order to illustrate where the main problem is, let us start with an Hilbert space different from the BvK one, using instead the boundary conditions which are appropriate for the bound states of an isolated atom or molecule. In this case the many-body wavefunction $\Phi_{0}\left(x_{1}, \ldots, x_{i}, \ldots, x_{N}\right)$ goes to zero exponentially whenever $\left|x_{i}\right|$ gets large. Within this Hilbert space there is no problem with the position, which is trivially defined as the multiplicative operator

$$
\hat{X}=\sum_{i=1}^{N} x_{i}
$$

within the Schrödinger representation. The ground-state expectation value is then simply:

$$
\langle X\rangle=\left\langle\Phi_{0}|\hat{X}| \Phi_{0}\right\rangle=\int d x x n(x)
$$

where $n(x)$ is the one-particle density. The value of $\langle X\rangle$ scales with the system size, and the quantity of interest is indeed the dipole per unit length, which coincides 
with macroscopic polarization. The expectation value in Eq. (3) is dominated by surface effects, thus making the definition of a bulk quantity problematic. If instead one adopts BvK, Eq. (1), then the operator defined by Eq. (2) is no longer a legitimate one, as explained above.

The major goal is therefore to define the expectation value of the electronic position $\langle X\rangle$ in the BvK Hilbert space, and to prove that our definition provides in the thermodynamic limit the physical macroscopic polarization of the sample: this has been very recently achieved in Ref. [19]. Before illustrating this major advance, we discuss an important precursor work, apparently unrelated with the polarization problem, where nonetheless the expectation value of the position operator plays the key role.

\section{THE ELECTRON-IN-BROTH FORMULA}

Some years ago, A. Selloni et al. [20] addressed the properties of electrons dissolved in molten salts at high dilution, in a paper which at the time was commonly nicknamed the "electron in broth". The physical problem was studied by means of a mixed quantum-classical simulation, where a lone electron was adiabatically moving in a molten salt (the "broth") at finite temperature. The simulation cell contained 32 cations, 31 anions, and a single electron. $\mathrm{KCl}$ was the original case study, which therefore addressed the liquid state analogue of an F center; other systems were studied afterwards [23]. The motion of the ions was assumed as completely classical, and the Newton equation of motions were integrated by means of standard molecular dynamics (MD) techniques, though the ionic motion was coupled to the quantum degree of freedom of the electron. The electronic ground wavefunction was determined solving the time-dependent Schrödinger equation at each MD time step. Two snapshots of the simulation, reproduced from the original paper, are shown in Fig. 1.

As usual in MD simulations, periodic boundary conditions are adopted for the
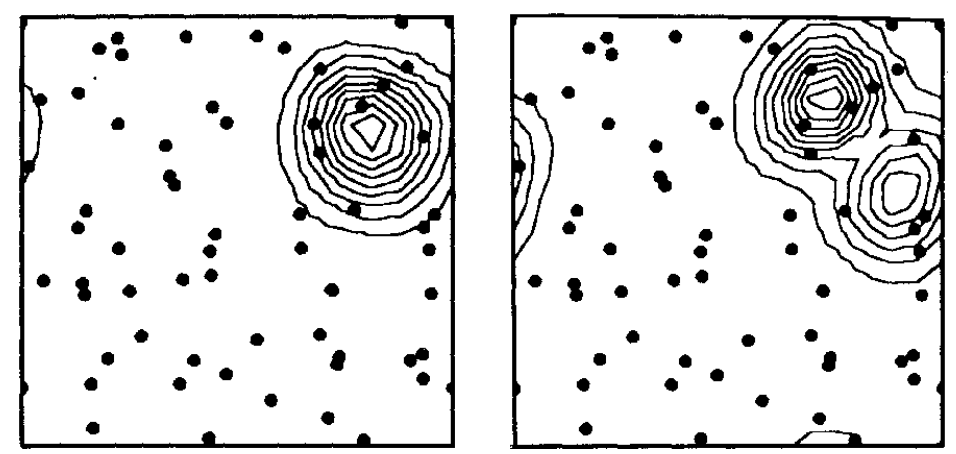

FIGURE 1. Contour plot of the electronic density (integrated along the sight line) at two different time steps. The dots are projections of the ionic positions. After Ref. [20]. 
classical ionic motion. Ideally, the ionic motion occurs in a simulation cell which is surrounded by periodic replicas: inter-cell interactions are accounted for, thus avoiding surface effects. Analogously, the electronic wavefunction is chosen in the work of Selloni et al. to obey BvK over the simulation cell, and therefore features periodic replicas as well. In Fig. 1, the "center" of the electron distribution is close to one of the borders of the simulation cell: the contour plot clearly shows the tail of its replica spilling into the opposite border.

One of the main properties investigated in Ref. [20] was the electronic diffusion, where the thermal ionic motion is the driving agent (within the adiabatic approximation). In order to perform this study, one has to identify first of all where the "center" of the electronic distribution is. Intuitively, the distributions in Fig. 1 appear to have a "center", which however is defined only modulo the replica periodicity, and furthermore cannot be evaluated simply as $\int d \mathbf{r} \mathbf{r}|\psi(\mathbf{r})|^{2}$ precisely because of BvK. Selloni et al. solved the problem by means of a very elegant and far-reaching formula. Here I write its one-dimensional analogue in the form:

$$
\langle x\rangle=\frac{L}{2 \pi} \operatorname{Im} \log \int_{0}^{L} d x \mathrm{e}^{i \frac{2 \pi}{L} x}|\psi(x)|^{2} .
$$

This formula provides the center of the electronic distribution, which is defined unambiguously though modulo $L$, as it must be.

\section{HOW THE FORMULA WORKS}

The density of the single-particle wavefunction $n(x)=|\psi(x)|^{2}$ obeys BvK periodicity and can therefore be written as a Fourier series:

$$
n(x)=\sum_{s=-\infty}^{\infty} C_{s} \mathrm{e}^{i \frac{2 \pi s}{L} x}
$$

The electron-in-broth formula, Eq. (4) becomes:

$$
\langle x\rangle=\frac{L}{2 \pi} \operatorname{Im} \log \int_{0}^{L} d x \mathrm{e}^{i \frac{2 \pi}{L} x} n(x)=\frac{L}{2 \pi} \operatorname{Im} \log C_{-1},
$$

a quantity which is well defined (modulo $L$ ) whenever the $s=-1$ Fourier coefficient is nonvanishing.

In general, any periodic function $n$ can be written - in a nonunique way - as the superposition of a localized function $n_{\mathrm{loc}}$ and of its periodic replicas. We write in the most general case the superposition as

$$
n(x)=\sum_{n=-\infty}^{\infty} n_{\mathrm{loc}}\left(x-x_{0}-n L\right)
$$

where $x_{0}$ is hitherto arbitrary. Then the Fourier coefficients of $n(x)$ are related to the Fourier transform of $n_{\mathrm{loc}}(x)$ as: 


$$
C_{s}=\frac{1}{L} \tilde{n}_{\mathrm{loc}}\left(\frac{2 \pi s}{L}\right) \mathrm{e}^{-i \frac{2 \pi s}{L} x_{0}} .
$$

We therefore express $\langle x\rangle$, Eq. (4), as:

$$
\langle x\rangle=\frac{L}{2 \pi} \operatorname{Im} \log \left[\mathrm{e}^{i \frac{2 \pi}{L} x_{0}} \tilde{n}_{\mathrm{loc}}\left(-\frac{2 \pi}{L}\right)\right]=x_{0}+\frac{L}{2 \pi} \operatorname{Im} \log \tilde{n}_{\mathrm{loc}}\left(-\frac{2 \pi}{L}\right) .
$$

We now specialize to the only interesting case: $n_{\mathrm{loc}}(x)$ is a real positive function, which integrates to one over $(-\infty, \infty)$; furthermore $n_{\text {loc }}(x)$ is centered at $x=0$ and decays over distances of the order of $\Delta x$. In the special case where $n_{\text {loc }}(x)$ is an even function, its Fourier transform is real and Eq. (9) simplifies to $\langle x\rangle \equiv x_{0}$ $(\bmod L)$, which is indeed the expected result. In the general case where $n_{\text {loc }}(x)$ is not an even function, an expansion of its Fourier transform is needed. The Fourier transform $\tilde{n}_{\text {loc }}(k)$ decays over a reciprocal distance of the order of $1 / \Delta x$ : therefore supposing $\Delta x \ll L$ (as e.g. is clearly the case in Fig. 1) the expansion yields:

$$
\tilde{n}_{\mathrm{loc}}\left(-\frac{2 \pi}{L}\right)=\int_{-\infty}^{\infty} d x n_{\mathrm{loc}}(x)+i \frac{2 \pi}{L} \int_{-\infty}^{\infty} d x x n_{\mathrm{loc}}(x)+\mathcal{O}\left(L^{-2}\right) .
$$

Since by hypothesis $n_{\text {loc }}(x)$ integrates to one, Eq (9) to leading order yields:

$$
\langle x\rangle \simeq x_{0}+\int_{-\infty}^{\infty} d x x n_{\mathrm{loc}}(x) \quad(\bmod L)
$$

again a very meaningful expression for the center of the electronic distribution $n(x)$.

\section{POLARIZATION AS A MANY-ELECTRON PROBLEM}

In the previous two Sections we have discussed how to define the expectation value of the position operator within BvK, when only a single electron is present in a large cell. When dealing instead with macroscopic polarization, we are faced with a system of many electrons, having a finite density. As anticipated above, our main interest is in studying a bulk system: $N$ electrons in a segment of length $L$. Eventually the thermodynamic limit is taken: $L \rightarrow \infty, N \rightarrow \infty$, and $N / L=n_{0}$ constant. If one arrives at defining a suitable position expectation value $\langle X\rangle$, analogue of Eq. (3) for the much less trivial BvK case, then the electronic contribution to macroscopic polarization (dipole per unit length) is:

$$
P_{\mathrm{el}}=\lim _{L \rightarrow \infty} \frac{e\langle X\rangle}{L}
$$

where $e$ is the electron charge.

So the main issue is as follows: can the electron-in-broth formula, Eq. (4), be generalized to provide an expectation value $\langle X\rangle$ appropriate to the case of many electrons in the given BvK periodicity? The answer is yes, but a smart generalization is needed. The most straightforward generalization, where one simply 
identifies $n(x)$ with the electron density of the extended system, does not work at all. There is a very good reason for this.

Suppose the system is crystalline, and furthermore assume independent electrons for the sake of symplicity. According to the modern viewpoint [7] the macroscopic polarization is a bulk observable completely independent from the periodic charge distribution of the polarized dielectric. While the density is obtained from the square modulus of the Bloch orbitals, polarization can only be obtained from their phase. Indeed, if one attempts to actually evaluating Eq. (4) upon replacement of $|\psi(x)|^{2}$ with the bulk density $n(x)$ of the extended system a meaningless result is reached. In fact, if the lattice constant of the crystalline solid is $a$, the BvK periodicity is then taken over a multiple of $a$ : $L=M a$. Since $n(x)$ is periodic with period $a$, its Fourier coefficients $C_{s}$ in Eq. (5) vanish unless $s$ is a multiple of $M$ : therefore $C_{-1}=0$, and the electron-in-broth formula, Eq. (6), is ill defined.

In order to arrive at the correct many-electron generalization of Eq. (4), one first introduces the multiplicative many-body operator $\mathrm{e}^{i \frac{2 \pi}{L} \hat{X}}$, where $\hat{X}$ is the simple operator defined in Eq. (2). In terms of this, one then defines:

$$
\langle X\rangle=\frac{L}{2 \pi} \operatorname{Im} \log \left\langle\Psi_{0}\left|\mathrm{e}^{i \frac{2 \pi}{L} \hat{X}}\right| \Psi_{0}\right\rangle .
$$

The similarity with Eq. (4) is self evident; as in the one-body case, the position expectation value is defined only modulo $L$. The main ingredient of our generalized electron-in-broth formula is a many-body expectation value: an $N$-electron integral where a genuine many-body operator appears. In general, one defines an operator to be one-body whenever it is the sum of $N$ identical operators, acting on each electronic coordinate separately: for instance, the $\hat{X}$ operator itself is such. In order to express the expectation value of a one-body operator the full many-body wavefunction is not needed: knowledge of the reduced one-body reduced density matrix $\rho$ is enough: this is e.g. the case in Eq. (3). I stress that, instead, the expectation value of $\mathrm{e}^{i \frac{2 \pi}{L} \hat{X}}$ over a correlated wavefunction cannot be expressed in terms of $\rho$, and knowledge of the $N$-electron wavefunction is explicitly needed. The uncorrelated case where $\left|\Psi_{0}\right\rangle$ is a single determinant will be discussed in the next Section.

The electronic polarization, Eq. (12), becomes then:

$$
P_{\mathrm{el}}=\lim _{L \rightarrow \infty} \frac{e}{2 \pi} \operatorname{Im} \log \left\langle\Psi_{0}\left|\mathrm{e}^{i \frac{2 \pi}{L} \hat{X}}\right| \Psi_{0}\right\rangle .
$$

As anticipated in the Introduction, this compact and general expression for the macroscopic polarization applies on the same footing to correlated systems and to independent-electron systems, as well as to crystalline and to disordered systems. Notice that in any case $L \rightarrow \infty$ is an highly nontrivial limit, since the exponential operator in Eq. (14) goes formally to the identity, but the size of the system and the number of electrons in the wavefunction increase with $L$. Therefore some $a d-h o c$ procedure is needed to demonstrate Eq. (14): this is reported in detail in Ref. [19], and will not be repeated here. 


\section{INDEPENDENT PARTICLES AND SINGLE-POINT BERRY PHASE}

We focus here on an uncorrelated system of independent electrons, whose $N$ electron wavefunction $\left|\Psi_{0}\right\rangle$ is a Slater determinant. In this special case $\left|\Psi_{0}\right\rangle$ is uniquely determined by the one-body reduced density matrix $\rho$ (which is the projector over the set of the occupied spinorbitals): therefore the expectation value $\langle X\rangle$, Eq. (13), is uniquely determined by $\rho$. The expression for $\langle X\rangle$-as well as the related one for the electronic polarization - is however most simply expressed directly in terms of the orbitals, as I am going to show. Such an expression, which goes under the name of "single-point Berry phase", was first proposed in a series of lecture notes [21]; it has been further scrutinized in some detail in a recent paper by Yaschenko et al. [22].

Suppose $N$ is even, and $\left|\Psi_{0}\right\rangle$ is a singlet. The Slater determinant has thus the form:

$$
\left|\Psi_{0}\right\rangle=\frac{1}{\sqrt{N !}}\left|\varphi_{1} \bar{\varphi}_{1} \varphi_{2} \bar{\varphi}_{2} \ldots \varphi_{N / 2} \bar{\varphi}_{N / 2}\right|
$$

where $\varphi_{i}$ are the single-particle orbitals. It is then expedient to define

$$
\left|\tilde{\Psi}_{0}\right\rangle=\mathrm{e}^{i \frac{2 \pi}{L} \hat{X}}\left|\Psi_{0}\right\rangle:
$$

even $\left|\tilde{\Psi}_{0}\right\rangle$ is indeed a Slater determinant, where each orbital $\varphi_{i}(x)$ of $\left|\Psi_{0}\right\rangle$ is multiplied by the plane wave $\mathrm{e}^{i \frac{2 \pi}{L} x}$. According to a well known theorem, the overlap amongst two determinants is equal to the determinant of the overlap matrix amongst the orbitals. We therefore define the matrix (of size $N / 2 \times N / 2$ ):

$$
S_{i j}=\int_{0}^{L} d x \varphi_{i}^{*}(x) \mathrm{e}^{i \frac{2 \pi}{L} x} \varphi_{j}(x)
$$

in terms of which we easily get

$$
\langle X\rangle=\frac{L}{2 \pi} \operatorname{Im} \log \left\langle\Psi_{0} \mid \tilde{\Psi}_{0}\right\rangle=\frac{L}{\pi} \operatorname{Im} \log \operatorname{det} S,
$$

where the factor of 2 accounts for spin.

Notice that nowhere we have assumed crystalline symmetry: the $\varphi_{i}$ orbitals will therefore have the most general form, while obviously obeying BvK at the sample boundary. Eq. (18) applies on the same ground to crystalline and to disordered systems: in the crystalline case it can be shown to be equivalent to a discretization of the usual continuum Berry phase [7], where crystalline Bloch orbitals are used. A simple test case is studied by Yaschenko et al. [22], where the numerical equivalence is actually demonstrated.

The single-point Berry phase has been recently implemented quite successfully to calculate infrared spectra of disordered systems [24,25]. In a typical Car-Parrinello 
simulation [26], the thermodynamic limit is approximated using BvK over a large simulation cell, which contains several tens of atoms. The time evolution of the ions is followed along discrete time steps, while the electrons follow adiabatically. In order to evaluate the infrared spectrum, one needs to evaluate the electronic macroscopic current traversing the simulation cell. This is done on the fly with the use of the single-point Berry phase, where $\langle X\rangle$, Eq. (18), is evaluated at each time step, and its time derivative is approximated with a finite difference [24].

\section{CONCLUSIONS}

The formula originally proposed by Selloni et al. [20] for dealing with a lone electron in a large liquid sample, and named here the "electron-in-broth formula", is quite naturally generalized to the many-body case. It provides then a novel solution to the polarization problem, Eq. (14): such solution, though apparently unrelated to the Berry-phase concept, is indeed a discretized Berry phase in disguise [19].

Since any Berry phase is by definition a phase, it may appear surprising that in Eq. (14) only the square modulus of the wavefunction enters: any phase information is then apparently obliterated. One has to bear in mind, however, that $\left|\Psi_{0}\right\rangle$ is the many-body wavefunction of the $N$-electron system. In the independent-electron case, this wavefunction embeds the relevant information about the relative phases of the one-electron orbitals, and this is what one needs in order to evaluate the macroscopic polarization; in the correlated-electron case, the wavefunction carries in a more complex way the analogue information.

\section{ACKNOWLEDGMENTS}

Discussions with M. Bernasconi and E. Yaschenko are gratefully acknowledged. Work partly supported by the Office of Naval Research, through grant N00014-961-0689.

\section{REFERENCES}

1. L.D. Landau and E.M. Lifshitz, Electrodynamics of Continuous Media (Pergamon Press, Oxford, 1984).

2. C. Kittel, Introduction to Solid State Physics, 7th. edition (Wiley, New York, 1996).

3. N.W. Ashcroft and N.D. Mermin, Solid State Physics (Saunders, Philadelphia, 1976).

4. R.M. Martin, Phys. Rev. B 9, 1998 (1974).

5. R. Resta, Ferroelectrics 136, 51 (1992).

6. R. Resta, Europhys. Lett. 22, 133 (1993).

7. R. Resta, Rev. Mod. Phys. 66, 899 (1994).

8. R.D. King-Smith and D. Vanderbilt, Phys. Rev. B 47, 1651 (1993).

9. M.V. Berry, Proc. Roy. Soc. Lond. A 392, 45 (1984). 
10. Geometric Phases in Physics, edited by A. Shapere and F. Wilczek (World Scientific, Singapore, 1989).

11. R. Resta, Ferroelectrics, 151, 49 (1994).

12. R.M. Martin and G. Ortíz, Solid State Commun. 102, 121 (1997).

13. R. Resta, Europhysics News 28, 18 (1997).

14. Theory of the Inhomogeneous Electron Gas, edited by S. Lundqvist and N.H. March (Plenum, New York, 1983).

15. G. Ortíz and R.M. Martin, Phys. Rev. B 43, 14202 (1994).

16. R. Resta and S. Sorella, Phys. Rev. Lett. 74, 4738 (1995).

17. G. Ortíz, P. Ordejón, R.M. Martin, and G. Chiappe, Phys. Rev. B 54, 13515 (1995).

18. R. Resta, Ferroelectrics 194, 1 (1997).

19. R. Resta, Phys. Rev. Lett., in press (preprint cond-mat/9709306).

20. A. Selloni, P. Carnevali, R. Car, and M. Parrinello, Phys. Rev. Lett. 59, 823 (1987).

21. R. Resta, Berry Phase in Electronic Wavefunctions, Troisième Cycle Lecture Notes (Ecole Polytechnique Fédérale, Lausanne, Switzerland, 1996). Available online (194K) at the URL:

http://ale2ts.ts.infn.it:6163/ ${ }^{\sim}$ resta/publ/notes_trois.ps.gz.

22. E. Yaschenko, L. Fu, L Resca, and R. Resta, Phys. Rev. B, in press.

23. E.S. Fois, A. Selloni, M. Parrinello, and R. Car, J. Phys. Chem. 92, 3268 (1988); F. Ancilotto and F. Toigo, Phys. Rev. B 45, 4015 (1992).

24. M. Bernasconi and M. Parrinello, unpublished; A. Debernardi, M. Bernasconi, M Cardona, and M. Parrinello, Appl. Phys. Lett. 71, 2692 (1997); P.L. Sivestrelli, M. Bernasconi and M. Parrinello, Chem. Phys. Lett. 277, 478 (1997).

25. A. Pasquarello and R. Car, Phys. Rev. Lett. 79, 1766 (1997).

26. R. Car and M. Parrinello, Phys. Rev. Lett. 55, 2471 (1985). 